\title{
CONCEPCIONES ALTERNATIVAS EN FÍSICA Y QUÍMICA DE BACHILLERATO: UNA METODOLOGÍA DIAGNÓSTICA
}

\author{
VÁZQUEZ ALONSO, A. \\ Servicio de Inspección Técnica de Educación. Palma de Maltorca.
}

\section{SUMMARY}

This paper emphasizes the independence between alternative conceptions and the type of instrument applied to identify them, as well as the usefulness of quantitative criteriums suggested in asses them.

\section{CONCEPCIONES ALTERNATIVAS EN \\ FÍSICA Y QUÍMICA DE BACHILLERATO: UNA METODOLOGÍA DIAGNOSTICA}

\begin{abstract}
El estudio de las concepciones alternativas en ciencias (errores conceptuales, ciencia de los niños, ideas previas, concepciones espontáneas...) se ha convertido en un tópico robusto de la investigación empírico-didáctica de la enseñanza-aprendizaje de las ciencias. Las páginas de ésta y otras revistas especializadas han sido testigos en estos últimos años de los principales hallazgos en torno al tema, por lo que resulta ocioso repetir los numerosos artículos aparecidos. Si acaso, por lo reciente de su aparición y la visión sintética que ofrece del tema (en el área de Física y Química) se puede citar el libro de Hierrezuelo y Montero (1989), que permite una rápida puesta al día de los no iniciados. En cuanto a la terminología, se empleará la denominación de concepciones alternativas compartiendo los argumentos en su favor defendidos por Abimbola (1988). Como características principales de las concepciones alternativas se admiten su persistencia, su coherencia interna con el sistema mental de cada inđividuo, y su amplia extensión de muestras en todas las edades y niveles culturales.
\end{abstract}

Sin embargo, la importancia y consistencia de estos rasgos tan universales puede quedar devaluada, entre los profesores de ciencias, por la sofisticación percibida de algunos métodos empleados en su detección.

\section{PLANTEAMIENTO DEL PROBLEMA}

Es bien conocido que la investigación sobre concepciones alternativas se ha centrado en la identificación de los errores más comunes cometidos por los alumnos cuando se les cuestiona, directamente, la idea bajo estudio. La forma en que se hace(n) la(s) pregunta(s) ha sido muy variada (oral, escrita o manipulativa); las entrevistas abiertas, el diálogo «socrático», las discusiones sobre ejemplos y fenómenos, las entrevistas clínicas (piagetianas), las respuestas escritas, abiertas o cerradas, y el dibujo de diagramas o esquemas han sido métodos utili- 
zados frecuentemente en la identificación. Es importante resaltar, como denominador común en la detección de las concepciones alternativas, la aplicación de una metodología específica para esa investigación, que tal vez puede percibirse alejada de la actividad habitual de enseñanza-aprendizaje desarrollada en las aulas por el común de los profesores. En efecto, cuando se analizan algunas de las pruebas o ejemplos utilizados en las investigaciones de concepciones alternativas, $\mathrm{y} / \mathrm{o}$ algunas lecturas o interpretaciones que los investigadores realizan de las respuestas abiertas, el lector no iniciado puede percibir una cierta sofisticación o excesiva artificiosidad, que le pueden llevar a la falsa impresión superficial de que las concepciones alternativas sólo aparecen cuando una prueba «ad hoc» se prepara para detectarlas. Dicho con otras palabras, las concepciones alternativas se podrían percibir como un resultado originado, parcialmente, por la metodología usada (instrumentos, interpretación, etc.), y, en consecuencia, concluir que no conciernen directamente a la tarea como profesor (director del cambio conceptual de sus alumnos).

Parece útil plantear una tesis reivindicativa de lo obvio: las concepciones alternativas son independientes del método empleado en su detección; complementariamente, las concepciones alternativas se hacen patentes también en cualquier método de evaluación habitual en las clases de ciencias. Como apoyo de esta hipótesis, este artículo pretende evidenciar la aparición de concepciones alternativas en las respuestas de los alumnos a cuestiones ordinarias y habituales en la práctica escolar del proceso de enseñanza-aprendizaje de la Física y Química. Por otro lado, se pretende enfatizar la impor* tancia instrumental y metodológica de las pruebas objetivas de elección múltiple, no sólo en la evaluación de alumnos (avalada por la gran tradición de su aplicación, sobre todo en países anglosajones), sino en la identificación diagnóstica de concepciones alternativas en grupos de alumnos. Este diagnóstico puede ser el primer paso para un planteamiento del proceso de enseñanza-aprendizaje que permita el cambio conceptual, y por ello, la construcción de significados que debe perseguir la enseñanza/aprendizaje de las ciencias como objetivo principal.

\section{METODOLOGÍA}

Se han utilizado tres pruebas objetivas de elección múltiple como instrumentos de diagnóstico inicial de los conocimientos de Física y Química en alumnos que comenzaban, respectivamente, segundo y tercero de BUP y COU. El contenido de las pruebas objetivas tomaba como referente los contenidos y objetivos centrales (core-currículo) del curso anterior al que se aplicaba la prueba objetiva; así, la prueba aplicada a alumnos de $2^{\circ}$ de BUP estaba centrada en el currículo de EGB (30 ítems), la prueba aplicada a alumnos de $3^{\circ}$ de BUP se basó en los contenídos de $2^{\circ}$ ( 45 ítems) y la prueba objetiva aplicada a alumnos de COU versaba sobre contenidos de $3^{\circ}$ de BUP (45 ítems). Se tomaron cuestiones habituales de los libros de texto y se les dió la forma de ítems de opción múltiple, con cuatro distractores y una, y sólo una, respuesta correcta. Las pruebas objetivas fueron construidas extrayendo los ítems de un banco amplio, previamente ensayados y baremados en una aplicación piloto, siguiendo la metodología de tipificación propia de las pruebas objetivas, y ampliamente descrita en otro lugar (Vázquez 1989). La validez de contenido de las pruebas fue confirmada, en una encuesta paralela, por una amplia muestra de profesores de los alumnos a quienes se administraron las pruebas objetivas.

En conclusión, las pruebas objetivas construidas están formadas por cuestiones cuyo contenido es habitualmente usado por los profesores en la evaluación de alumnos, y no por preguntas construidas específicamente para detectar concepciones alternativas; en particular, ningún distractor fue diseñado teniendo en cuenta apriorísticamente las posibles Cas compatibles con el ítem. Con ello se pretende demostrar que las concepciones alternativas emergen también cuando se evalúa a los alumnos mediante cuestiones comunes, y no específicas para detectar concepciones alternativas, e inventariar, brevemente, cuáles han sido las concepciones alternativas encontradas.

Para ilustrar con claridad las pautas que permiten la detección de concepciones alternativas mediante cuestiones habituales de evaluación en Física y Química (no específicas de concepciones alternativas) es necesario revisar algunos de los principios fundamentales de la construcción de pruebas objetivas de elección múltiple.

Un ítem está formado por un 'pie' o 'base' en el que se plantea la cuestión a responder, seguido por cierto número de posibles respuestas (en nuestro caso 5), de las cuales algunas son falsas (distractores, 4) y otra(s) correcta(s) (en nuestro caso, 1). La tarea del alumno consiste en señalar la respuesta correcta. El principio fundamental de la Teoría Clásica de Respuesta a este tipo de pruebas objetivas es que únicamante se puede afirmar con certeza absoluta que un alumno que yerra la respuesta, desconoce ésta, en tanto, que el acierto no necesariamente implica el conocimiento de la respuesta. Por tanto, esto da la certeza de que las tasas de respuesta observadas en los distractores (respuestas erróneas) pueden corresponder a concepciones alternativas.

Una buena prueba objetiva necesita que los distractores sean homogéneos, es decir, todos ellos deben parecer igualmente atractivos que la respuesta correcta para el alumno desinformado. En consecuencia, se espera que las tasas de respuesta a los distractores deben ser similares entre sí, y por tanto, las desviaciones fuertes de la homogeneidad en las tasas de respuesta en algún distractor se interpretan como debidas, o bien, a que el citado distractor no está bien diseñado (generalmente, distractores no elegidos porque es obvia su falsedad), o bien, a que la respuesta reflejada en él tiene una atracción superior a las demás para los alumnos (distractores elegidos por encima de los demás, e incluso, de la respuesta correcta). Este último caso correspondería a la existencia de respuestas erróneas ampliamente implantadas en los alumnos, y por tanto, se interpreta como signo de la posible evidencia de concepciones alternativas. 
En consecuencia, se ha utilizado el análisis de las respuestas a los distractores de una prueba objetiva como base para identificar posibles concepciones aitemativas en los alumnos, aplicando dos criterios:

1. Tasa de respuesta de un distractor superior a la tasa de respuesta obtenida por la alternativa correcta.

2. Tasa de respuesta de un distractor significativamente superior a las tasas de los otros distractores.

Los ítems que cumplan alguno de estos criterios empíricos pueden mostrar concepciones alternativas en los alumnos. Su aplicación en el análisis de las tasas de respuesta obtenidas en cada ítem de las pruebas objetivas ha permitido identificar algunas concepciones alternativas.

La muestra de alumnos empleada correponde a 8 institutos de bachillerato de la isla de Mallorca: 591 alumnos que comenzaban $2^{\circ}$ de BUP ( 16 años) contestaron la prueba de EGB; 355 alumnos que comenzaban $3^{\circ}$ (17 años) contestaron la prueba objetiva de Física y Química de $2^{\circ}$, y 334 alumnos que comenzaban COU (18 años) respondieron la prueba objetiva de Física y Química de $3^{2}$. Ninguno de los alumnos de la muestra era repetidor de curso.

\section{RESULTADOS}

Se han analizado las tasas de respuestas obtenidas en los 120 ítems que contenían las pruebas objetivas aplicadas; empleando los dos criterios explicitados anteriormente, se han identificado los ítems que muestran concepciones alternativas en los alumnos. El número de ítems encontrado que satisfacen alguno de los dos criterios aplicados (16 2) es notable, y mayor cuanto más elevado es el curso al que se refiere la prueba. Las tablas resumen las concepciones alternativas encontradas en las respuestas a los ítems de las pruebas objetivas mediante una descripción sucinta de ellas. Para que el lector pueda contrastarlas y analizarlas por sí mismo, se indican el ítem del que se han deducido y el criterio aplicado (1 6 6), estando el texto de la mayoría de los ítems (no todos) en los Apendices. Tanto en las tablas como en los Apéndices los resultados se han agrupado en tres bloques: Mecánica/Calor, Electricidad/Optica y Química. Cada ítem se describe con un número en clave de dos cifras separadas por un punto; la primera cifra indica el curso (2 para segundo, 3 para tercero y 4 para $C O U$ ), y la segunda es el número de orden del ítem en las pruebas objetivas de las que se han extraído. A veces, se incluye la codificación (MS) para indicar que, presumiblemente, algunos elementos de la llamada 'metodología de la superficialidad' (Carrascosa \& Gil 1985) han influenciado la respuesta.

Los resuitados refrendan muchas de las concepciones alternativas informadas en la bibliografía investigacio. nal sobre este tema, con la diferencia metodólogica fundamental que, en este estudio, la identificación de concepciones alternativas no era el objetivo principal de la aplicación de las pruebas objetivas, puesto que el proceso de redaccion y selección de los íterns no ha sido orientado por la detección de concepciones alternativas, sino sólo para realizar un diagnóstico del nivel instructivo inicial de los alumnos, en cada uno de los cursos estudiados. Los resultados obtenidos demuestran la independencia de la aparición de las concepciones alterna . tivas respecto al tipo de metodología o instrumento empleado en la evaluación: los errores identificados se corresponden con los informados en la amplia literatura sobre el tema. En particular, un instrumento de evaluación objetivo del tipo empleado aquí (prueba objetiva) permite la aplicación de criterios cuantificables y objetivos, y por ello, válidos y fiables, además de sencillos, que posibilitan su empleo por cualquier profesor de ciencias.

Por otro lado, para los alumnos mayores (alumnos de COU - fítems cuyo primer número es el 4- que responden cuestiones de Física y Química de $3^{\circ}$ de BUP) se observa un persistente aumento del número de concepciones alternativas que responden a la aplicación de la 'metodología de la superficialidad', es decir, respuestas acríticas elicitadas en muchos casos por distractores que 'suenan' bien, o contienen elementos que se identifican con las rutinas más mecánicas y estereotipadas adquiri. das en las clases de ciencias.

Este rasgo, sin duda, pone de relieve que el tipo de enseñanza/aprendizaje recibido por los alumnos dista mucho de estar de acuerdo con los elementos epistemológicos fundamentales de la ciencia y el modo de producción científico. Los alumnos mayores, que han ido adquiriendo en los sucesivos cursos más conocimientos científicos, evidencian paralelamente un aumento en $s u$ bagage de destrezas maladaptativas; en particular, los errores identificados con el nombre de metodología de la superficialidad (Carrascosa y Gil 1985). Parece pues, que la cantidad de información creciente no basta para propiciar el cambio conceptual, y por tanto, no consigue aprendizajes significativos, sino que se refuerzan conductas maladaptativas, estereotipadas y espúreas.

\section{CONCLUSIONES}

Los resultados evidencian que las mismas concepciones alternativas de los alumnos en ciencias son elicitadas por cualquier tipo de reactivo estimular (en este caso una prueba objetiva no diseñada para ello), y no sólo ante cuestiones ad hoc, preparadas especialmente para detec. tar estas concepciones alternativas. Diseñando pruebas objetivas sobre la base de una tabla de especificaciones integrada por concepciones alternativas sería posible su diagnosis sistemática en la formación científica de los altumnos.

Las pruebas objetivas aplicadas en este estudio, no diseñadas con finalidad diagnóstica para las concepcio- 
nes alternativas, han evidenciado la facilidad con que las concepciones altemativas aparecen y pueden evaluarse. Los criterios apificados en este estudio proveen un elemento cuantificado, objetivo y fiable que puede permitir la comparación entre estudios diferentes que apliquen estos mismos criterios, y en consecuencia, facilitan la comparación de resultados entre estudios, pruebas y

\section{REFERENCIAS BIBLIOGRÁFICAS}

ABIMBOLA, I.O., 1988. The Problem of Terminology in the Study of Student Conceptions in Science, Science Education, 72(2), pp. $175-184$.

CARRASCOSA ALIS, J. y GIL PÉREZ, D., 1985. La «metodología de la superficialitat» i l'aprenentatge de les ciències, Enseñanza de las Ciencias, 3, pp. 113-120. poblaciones diferentes. Por último, el aumento con Ios cursos del número de concepciones alternativas encontradas, así como los rasgos crecientes de la metodología de la superficialidad hallados, evidencian que los aprendizajes realizados no promueven el cambio conceptual $y$, posiblemente, están lejos de ser aprendizajes significativos.
HIERREZUELO, J. y MONTERO, A., 1989. La ciencia de los alumnos. (Ministerio de Ėducación-Laia: Barcelona).

VÁZQUEZ ALONSO, A., 1989. Rendimiento en Bachillerato: aptitudes y atribución causal. Analisis del rendimiento objetivo en Física y Química. Tesis Doctoral no publicada, Universidad de las Isias Baleares.

Tabia I

Concepciones alternativas de los alumnos de Bachilterato en Mecánica y Calor. A la derecha se indica el criterio aplicado para su identificación (texto de algunos items en el Apéndice I).

\begin{tabular}{|c|c|c|}
\hline \multicolumn{3}{|c|}{ MECÁNICA/CALOR } \\
\hline fiem & Concepción aitemativa & Criterio \\
\hline 3.14. & Confusión entre aceleración y veiocidad; identificación del m.u.a. con movimientos uniformes. & 1 \\
\hline 4.10. & No existe aceleración en el movimiento circular unifome. & 2 \\
\hline 3.4. & Las fuerzas se comportan como magnitudes escalares. & 2 \\
\hline 3.10 . & Independencia entre la aceleración y la masa. & 1 \\
\hline 4.13. & La existencia de aceleración no está limitada al intervalo de tiempo que dura la acción de una fuerza. & 1 \\
\hline 4.13. & El movimiento (uniforme) requiere la acción de una fuerza neta aplicada. & 1 \\
\hline 4.15. & El principio de conservación de la energía se ignora: la energia se gasta siempre. & 2 \\
\hline 4.3. & No se comprende el concepto de kilowatio-hora. & 1 (MS) \\
\hline 2.1 & La temperatura durante un cambio de estado no pemanect constante. & \\
\hline 2.1 . & La temperatura de canbio de estado es la máxima. & 2 \\
\hline 2.3. & Desconocimiento de la existencia de la convección y conducción conto mecanismos de transmisión đe calor. & 1 \\
\hline 3.3. & La temperatira para cambios de estado inversos no es la misma (fusión/solidificación ...). & 1 \\
\hline 3.15 & Incomprensión del concepto de calor específico. & 1 \\
\hline 3.8. & Proporcionalidad de la masa con la longitud, y no el volumen (cubo de la longitud). & 1 \\
\hline 3.17. & No se relaciona el empuje con la densidad del líquido que sustenta el sólido que flota. & 2 \\
\hline
\end{tabular}

(MS): metodología de la superficialidad. 
Tabla II.

Concepciones alternativas de los alumnos de Bachillerato en Electricidad y Optica. A la derecha se indica el criterio aplicado para su identificación (texto de algunos items en el Apéndice II).

\begin{tabular}{|c|c|c|}
\hline \multicolumn{3}{|c|}{ MECÁNICAJCALOR } \\
\hline$\underline{\text { ftem }}$ & Concepción alcemativa & Criterio \\
\hline 2.5 & Confusión entre intensidad de corriente y tensión a través de sus unidades. & 1 \\
\hline 3.5 . & No se comprende la dependencia con el cuadrado de la distancia para la ley de Coulomb (proporcionalidad simple). & 1 (MS) \\
\hline 3.11. & Confusión lingüistica entre intensidad de campo e intensidad de corriente. & 2 \\
\hline 3.16 . & Confusión aplicativa entre campo eléctrico y potencial eléctrico. & 1 \\
\hline 4.2 . & Confusión lingǘstica entre potencial e «intensidad electrica». & \\
\hline 4.8. & $\begin{array}{l}\text { La ley para el cálculo de la resistencia equivalente a una asociación en paralelo se toma referida a la resistencia } \\
\text { (no a su inverso) }\end{array}$ & 1 (MS) \\
\hline 2.6 & Ignorancia de la relación índice de refracción y velocidad de la luz (¿subyace su infinitud?). & 1 \\
\hline 2.7. & Realidad de una imagen especular. & 1 \\
\hline 2.7. & Confusión entre inversion arriba-abajo e intercambio derecha-izquicrda en los espejos. & 1 \\
\hline 3.9. & Confusión entre propagación de la luz con refracción y observación de un palo bajo el agua (doblado arriba). & 1 \\
\hline
\end{tabular}

(MS): metodología de la superficialidad

Tabla III.

Concepciones alternativas de los alumnos de Bachillerato en Química. A la derecha se indica el criterio aplicado para su identificación (texto de ajgunos ítems en el Apénơice III).

MECÁNICA/CALOR

\begin{tabular}{llll}
\hline Ítem Concepción alcemativa & Criterio \\
\hline
\end{tabular}

2.2. Confusión entre el número de átomos de una molécula, los subindices y el número de especies atómicas. 2

2.4. Los átomos de los sólidos están quietos; sólo se mueven los de los gases. 1

2.8. Confusion entre sustancia simple y átomo.

3.1. Concepto de disolución asociado con líquidos. 2

3.6. Confusión entre proporcionalidad directa e inversa. 1

3.7. Confusión entre mol y masa molecular. $\quad 1$

3.18. Confusion entre subindices y valencias en formulas. 2 (MS)

$\begin{array}{ll}\text { 3.12. Se elige la fórmula que más «suena” (glucosa...). } & \text { (MS) }\end{array}$

3.13. Identificacion de la cantidad de disolvente con la cantioad de disolución.

4.1. La asociación entre energía de ionización y clectropositiviơad, se hace al revés. 2 (MS)

4.6. Se confunde la convención para el número cuántico secundario $s=1, p=2, d=3 \ldots$, en lugar de $s=0, p=1, d=2$. I (MS)

4.9. El enlace covalente no se identifica por su definición, sino que es ensombrecido gor etiquetas que "sucnan" más. $\quad 1$ (MS)

$\begin{array}{ll}\text { 4.11. La molecula con mayor carăcter ínnico se identifica con la más habitual en los ejemplos (NaF). } & 1 \text { (MS) }\end{array}$

4.16. El enlace químico requiere la aportación del mismo número tolai de electrones por los átomos. 
Tabla III (Continuación)

\begin{tabular}{llc}
\hline ftem & Concepcion altemativa & Criterio \\
\hline $3.2 . \quad$ Metodología superficialidad sobre proposiciones de transformaciones REDOX. & 1 (MS) \\
$4.4 . \quad$ Los cazalizadores se emplean para aumentar la velocidad de las reacciones (no para disninuir). & 1 \\
$4.7 . \quad$ Se confunde el convenio de signos para el calor de reacción. & 1 (MS) \\
$4.12 . \quad$ La expresión de la constante de equilibrio reproduce por inetcia un estereotipo, sin tener en cuenta el estado & 1 (MS) \\
4.17. $\quad$ Elevando la temperatura se consigue siempre mayor eficiencia en totas las reacciones químicas. & 1 \\
\hline
\end{tabular}

\section{APÉNDICE I}

Texto de algunos f́tems que corresponden 8 las concepciones alternativas identificadas en Mecánica y Calor (Tabla I). Al final de cada ftem se recoge la tasa (\%) de respuestas por este orden: tasa de ausencia de respuesta y tasas correspondientes a cada altemativa del f́tem, en el mismo orden que guardan en el texto del íten;; aparece subrayado la respuesta correcta y con * las tasas más elevadas de distractores.

3.14. Un móvil parte đel reposo con aceleractón contante de $20 \mathrm{~cm} / \mathrm{s}^{2}{ }_{2} \mathrm{Cuánto}$ tiempo tardara en recorrer an kilometro?
A) $50 \mathrm{~s}$
B) $75 \mathrm{~s}$
C) $100 \mathrm{~s}$
D) $125 \mathrm{~s}$
E) $150 \mathrm{~s}$

*41.13. A)*26.48 $\quad$ B) 4.79 C) $18.03 \quad$ D) $5.07 \quad$ E) 4.51

4.10. Un móvil recorre una trayectoria circular de $5 \mathrm{~m}$ de radio con velocidad constante de $10 \mathrm{~m} \cdot \mathrm{s} \cdot 1$. El módulo de la aceleración total es (en m/s $\mathrm{s}^{2}$ ):
A) $v^{2} / R=10^{2} / 5$
B) $v / R=10 / 5$
C) Cero
D) $v \cdot R=10.5$
E) $v \cdot R^{2}=10.5^{2}$

14.37. A) 41.92 B) $4.76 \quad$ C) 25.75 D) 5.69 E) 7.49

3.4. Dos fuerzas concurrentes que están formando entre sí ángulo zecto, y valen 3 y $4 \mathrm{~N}$, tienen como fuerza resultante:
A) $7 \mathrm{~N}$
B) $1 \mathrm{~N}$
C) $4 \mathrm{~N}$
D) $3.5 \mathrm{~N}$
E) $5 \mathrm{~N}$

9.01. A)*23.38 $\quad$ B) 8.45 C) $3.10 \quad$ D) $6.76 \quad E) 49.30$

3.10. A un cuerpo de masa $m$ se aplica una fuerza $F$. A otro cuerpo de masa $m$ ' se le aplica la misma fuerza $F$ y resulta que la aceleración de $m$ es e) coble de la de $m^{\prime}$. Luego la masa $m$ vale:
A) $\mathrm{m}^{\prime}$
B) $2 m^{*}$
C) $4 m^{\prime}$
D) $m^{2} / 2$
E) $m * / 4$

4.13. Un cuerpo de $2 \mathrm{~kg}$ está en reposo sobre una superficie horizontal sin rozamiento; una fuerza constante de $2 \mathrm{~N}$ se aplica sobre el cuerpo, durante 3 segundos y se elimina. La velocidad del cuerpo a los $6 \mathrm{~s}$ será:
A) $1 \mathrm{~m} \cdot \mathrm{s} \cdot \mathrm{I}$
B) $3 \mathrm{~m} \cdot \mathrm{s}-1$
C) $6 \mathrm{~m} \cdot \mathrm{s}-1$
D) $12 \mathrm{~m} \cdot \mathrm{s}-1$
E) $24 \mathrm{~m} \cdot \mathrm{s} \cdot 1$

15.57. A) 7.78 B) $29.04 \quad$ C)*38.92 D) 5.99 E) 2.69

4.15. Desde el borde de una plataforma que está a cierta altura sobre el suelo se lanza un móvil hacia arriba con velociơad inicial. La anergfa mecánica totai es:
A) Mayor en el punto más alto que alcanza que los demás.
B) Minima en el momento de liegar al suelo.
C) Mayor en el nivel de la plataforma que en los demás.
D) Mínima en el nivel de la plataforma que en los demás.
E) La misma en todos los lugares que pasa.

$\begin{array}{llllll}* 21.26 \text {. A) } 14.97 & \text { B) } 7.78 & \text { C)*22.46 } & \text { D) } 3.29 & \text { E) } 30.24\end{array}$

4.3. Un kilowatio-hora es:
A) $3,6 \cdot 10^{6} \mathrm{I}$
B) $3,6 \cdot 10^{6} \mathrm{~N} / \mathrm{m}$
C) $1000 w / 3600 \mathrm{~s}$
D) $1000 \mathrm{I} / 3600 \mathrm{~s}$
E) $3,6 \cdot 10^{6} \mathrm{~W}$

11.08. A) 20.36 B) $.90 \quad$ C) $* 50.90$ D) $8.98 \quad$ E) 7.78

3.15. Un bloque de un metal $X$, de masa $m$ a $0^{\circ} \mathrm{C}$ se pone en contacto con otro bloque de un metal $Y$, de masa $2 m$ a $100^{2} \mathrm{C}$. Tiene lugar la transmisión de calor de uno a otro hasta alcanzar el equilibrio térmico los dos bloques a $20^{\circ} \mathrm{C}$. Si los calores específicos son $c_{x}$ y $c_{y}$, respectivamente, la relación entre los mismos es de :
A) $c_{x}=8 \cdot c_{y}$
B) $c_{x}=4 \cdot c_{y}$
C) $c_{x}=2+c_{y}$
D) $2 \cdot c_{x}=c_{y}$
E) $4 \cdot c_{x}=c_{y}$

$\begin{array}{lllll}* 61.13 . & \text { A) } 4.23 \quad \text { B)* }(1.83 & \text { C)* } 10.70 \quad \text { D)*7.32 E) } * 4.79\end{array}$

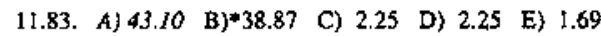


3.8. Tienes dos cubos de aluminio, el cubo $\mathrm{A}$ de $\mathrm{l} \mathrm{cm}$ de iado y el cubo B de $2 \mathrm{~cm}$ de lado. Podremos afirmar que:

A) La masa de A es la mitad de B, pero sus densidades son iguales.

B) La masa de B es 8 veces la de A y la densidad de B es 8 veces la de A.

C) La masa de $B$ es el doble que la de A y la densidad de $B$ es igual que la de A.

D) La masa de B es 8 veces la de A y las densidades de ambos son iguales.

E) La masa de B es el triple que la de A y las densidades son iguales.
3.17. Una pieza de madera flota sumergida totalmente en agua. Cuando se la coloca en alcohol (de densidad menor que el agua) ocurirá que:
A) Se hundirá en el fondo.
B) Flotará sobresaliendo más.
C) Quedará como antes.
D) Flotará, pero más sumergida.
E) Se hundirá un momento y luego flotará.

$\begin{array}{lllll}\text { *23.38. A) } 29.30 & \text { B)*16.90 } & \text { C) } .85 & \text { D) } * 22.82 & \text { E) } 6.76\end{array}$

$\begin{array}{lllll}\text { 18.31. A) } * 25.63 & \text { B) } 4.51 \quad \text { C)*30.99 D) } 14.37 & \text { E) } 6.20\end{array}$

\section{APÉNDICE 【}

Texto de algunos íterns que corresponden a las concepciones alternativas identificadas en Electricidad y Óptica (Tabla II). Al final de cada item se recoge la tasa (\%) de respuestas por este orden: tasa de ausencia de respuesta y tasas comespondientes a cada altenativa del íten, en el mismo orden que guardan en el texto del ítem; aparece subrayada la respuesta correcta y con * las tasas más elevadas de distractores.

2.5. La unidad de intensidad de la corriente eléctrica es:
A) El Culombio
B) El Voitio
C) El Ohmio
D) El Amperio
E) El Julio

2.54. A) 2.54 B)*57.36 C) 4.40 D) $31.30 \quad$ E) 1.86

3.5. Dos cargas eléctricas puntales están separadas entre sí $3 \mathrm{~m}$, repeliéndose con una fuerza de $10 \mathrm{~N}$. Si se separan $6 \mathrm{~m}$, la fuerza de repulsión será:
A) $2,5 \mathrm{~N}$
B) Dos veces menor
C) Dos veces mayor
D) $10 / 3 \mathrm{~N}$
E) $40 \mathrm{~N}$

9.58. A) $6.20 \quad$ B)*58.59 $\quad$ C) 17.18 D) $7.89 \quad$ E) .56
3.11. La uridad adecuada para la medida de la intensidad del campo eléctrico en un punto es:
A) C.m2
B) A-m
C) $\mathrm{N} / \mathrm{C}$
D) $\mathrm{m} / \mathrm{N}$
E) $\mathrm{A} / \mathrm{m}$

*35.21. A) 6.48 B) 12.39 C) 23.10 D) $2.82 \quad$ E)*20.00

4.8. La resistencia equivalente de dos resistencias asociadas en paralelo es:
A) El producto de las resistencias dividido por su suma.
B) La suma de las resistencias dividida por su producto.
C) La suma de los inversos de las resistencias.
D) El producto de los inversos de las dos resistencias.
E) La diferencia de los inversos de las dos resistencias.
7.78. A/ 13.78
B) $1.80 \quad$ C)*75.75
D) 2.69
E) 1.20

2.7. Las imágenes producidas por lentes, espejos o instrumentos 6 pticos pueden ser reales o virtuales. La imagen producida por un espejo es....
A) Siempre real
B) Unas veces real y otras virtual.
C) Siempre virtual.
D) Siempre derecha y más grantde.
E) Siempre invertida.

5.41. A)*22.17 B)*16.24 C) 22.50 D) 1.69 E)*31.98

\section{APENDICE III}

Texto de algunos ítems que corresponden a las concepciones aitemativas identificadas en Química (Tabla III). Al final de cada ítem se recoge la tasa (\%) de respuestas por este orden: tasa de ausencia de respuesta y tasas correspondientes a cada alternativa del item, en el mismo orden que guardan en el texto del íten; aparece subrayada la respuesta correcta y con * las tasas más elevadas de distractores.
2.2. Las moléculas de cualquier sustancia están formadas por la unión de varios átomos. De las que siguen señala la molécula triatómica:
A) $\mathrm{H}_{2} \mathrm{O}$
B) $\mathrm{Cl}_{2}$
C) $\mathrm{H}_{2} \mathrm{SO}_{4}$ 
D) $\mathrm{NO}$

E) $\mathrm{SO}_{3}$

13.54. A) 32.83
B) 3.55
C) $* 18.61$
D) 3.38 E) $* 28.09$

2.4. Los átomos de un cueppo sólido como el cobre oel hielo a la temperatura ambiental están...:
A) En continuo movimiento vibratorio alrededor de unas posiciones determinadas.
B) Quietos mientras sea sólido.
C) En continuo movimiento.
D) Solo moviéndose en estado gaseoso.
E) Quietos y nunca se mueven ya que el cobre y el hierro son muy pesados.
7.78 .
A) 26.57 B) $* 34.35$
C) $* 17.77$
D) 5.58
E) 7.95

2.8. En la relación siguiente tienes símbolos de varios elementos qufmicos y formulas de distintas sustancias; serala la formula que corresponde a una sustancia simple:
A) $\mathrm{Cl}$
B) $\mathrm{H}_{2} \mathrm{O}$
C) $\mathrm{O}$
D) $\mathrm{Cl}_{2} \mathrm{O}$
E) $\mathrm{N}_{2}$

$\begin{array}{llllll}3.05 & \text { A) } * 17.26 & 9.64 & \text { B) } * 54.99 & \text { C) } .17 & \text { D) } E / 14.89\end{array}$

3.6. ¿Cuál de las siguientes masas contiene el mismo número de átomos que 12 $g$ de magnesio? (Masas atónicas $\mathrm{C}=12, \mathrm{Ca}=40, \mathrm{Mg}=24$ )
A) $12 \mathrm{~g}$ de C
B) $20 \mathrm{~g}$ de $\mathrm{Ca}$
C) $24 \mathrm{~g} \mathrm{de} \mathrm{C}$
D) $40 \mathrm{~g} \mathrm{de} \mathrm{Ca}$
E) Ninguno de los anteriores.

$\begin{array}{llllll}\text { 16.62. } & \text { A) } 7.04 & \text { B) } 18.03 & \text { C) } * 40.56 & \text { D) } .56 & \text { E) } 17.18\end{array}$

3.7. El análisis de cierta sustancia muestra que tiene combinados $7 \mathrm{~g}$ de nitrogeno con $1 \mathrm{~g}$ de hidrógeno (Masas àónicas $\mathrm{N}=14, \mathrm{H}=1$ ). La fórmula más sencilla que cabe deducir para esta sustancia es:
A) $\mathrm{NH}_{2}$
B) $\mathrm{NH}_{7}$
C) $\mathrm{N}_{2} \mathrm{H}$
D) $\mathrm{N}_{2} \mathrm{H}_{4}$
E) $\mathrm{N}_{7} \mathrm{H}$
*21.41. A)
A) 22.25
B) $* 32.96$
C) 6.76
D) 2.54
E) 14.08

3.12. ¿Cuál de las siguientes fómmulas empiricas corresponde al benceno?
A) $\mathrm{C}_{6} \mathrm{H}_{6}$
B) $\mathrm{C}_{6} \mathrm{H}_{12}$
C) $\mathrm{C}_{6} \mathrm{H}_{10}$
D) $\mathrm{C}_{6} \mathrm{H}_{14}$
E) $\mathrm{C}_{6} \mathrm{H}_{3}$

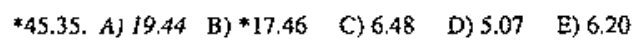

4.6. Un electrón de un atomo cuyos nümeros cuánticos principal y secundario son 4 y 2 , respectivamente, pertenece $a$ :
A) Nivel 2 y orbital s
B) Nivel 4 y orbital
C) Nivel 4 y orbitals

D) Nivel 6 y orbital d

E) Nivel 4 y orbital d

16.17. $\begin{array}{lllll}\text { A) } 9.58 & \text { B) } * 45.81 & \text { C) } 21.86 & \text { D) } 1.20 & \text { E) } 5.39\end{array}$

4.9. En la molécula de hidrógeno, los dos núcleos de los átomos se mantienen unidos entre sí por ....
A) Enlace iónico.
B) Compartición de la nube electrónica.
C) Enlace covalente dativo.
D) Atracción por fuerzas de Van der Waals.
E) Arracción intermolecular mediante enlaces por puente de hidrogeno.

12.57. A)*21.86 B) 13.47 C) $* 30.24 \quad$ D) $6.29 \quad$ E) $* 15.57$

4.11. Entre los siguientes compuestos señala aquel cuyo enlace presente mayor carácter iónico:
A) $\mathrm{RbCl}$
B) $\mathrm{NaF}$
C) $\mathrm{MgO}$
D) CsI
E) $\mathrm{CsF}$

*20.06. $\begin{array}{lllll}\text { A) } 7.78 & \text { B)*36.53 } & \text { C) } 9.58 & \text { D) } 7.19 & \text { E) } 18.86\end{array}$

4.16. Un elemento $X$ tiene 2 electrones de valencia y otro elemento $Z$ tiene 6 electrones de valencia. El compuesto que ambos pueden fomar al unirse quimicamente será probablemente:
A) Un compuesto iónico de fórmula $X Z$.
B) Un compuesto iónico de fórmula $X_{3} Z$.
C) Un compuesto iónico de fórmula $X_{2} Z_{2}$.
D) Un compuesto covalente XZ.
E) Un compuesto covalente de fórmula $X_{3} Z$.

14.07. A) 10.78 B) $* 33.23$ C) 2.99 D) 6.29 E) $* 32.63$

4.4. ¿Cuál de las siguientes frases explica mejor la acción de un catalizador en una reacción quínica?
A) Aumenta la velocidad de reacción.
B) Inicia la reacción.
C) Aumenta la energía de activación.
D) Aitera la velocidad de reacción.
E) Disminuye el calor de reacción.

9.28. A)*44.91 $\quad$ B) 3.59 C) $4.79 \quad 0) 30.84$ E) 6.59

4.12. ¿Cuál de las siguientes expresiones es la más correcta para indicar la constante de equilibrio del sistema:

$\mathrm{Cu}(\mathrm{s})+2 \mathrm{Ag}^{+}(\mathrm{ac}) \rightleftarrows \mathrm{Cu}+2 \mathrm{~s}(\mathrm{ac})+2 \mathrm{Ag}(\mathrm{s})$

A) $\mathrm{K}=[\mathrm{Cu}][\mathrm{Cu}+2] /\left[\mathrm{Ag}^{+}\right] \cdot[\mathrm{Ag}]$

B) $\mathrm{K}=\left[\mathrm{Ag}^{+}\right] /\left[\mathrm{Cu}^{2+}\right]$

C) $\mathrm{K}=\left[\mathrm{Cu}^{2+}\right] /\left[\mathrm{Ag}^{+}\right]$

D) $\mathrm{K}=\left[\mathrm{Ag}^{+}\right] /\left[\mathrm{Cu}^{2+}\right]$

E) $\mathrm{K}=[\mathrm{Ag}] /[\mathrm{Cu}]$

$\begin{array}{llllll}* 42.81 & \text { A)*27.25 } & \text { B) } 6.89 & \text { C) } 14.67 & \text { D) } 4.79 & \text { E) } 3.59\end{array}$ 